\title{
Reflexionando sobre la didáctica: un ciclo de mejora docente en la asignatura "Metodología y enseñanza de la lengua y literatura italiana"
}

\section{Reflecting on didactics: an improvement cycle in the subject "Methodology and teaching of Italian language and literature"}

DANIELE CERRATO

Universidad de Sevilla

Departamento de Filologías Integradas

dcerrato@us.es

ORCID: https://orcid.org/0000-0001-7238-1381

DOI: http://dx.doi.org/10.12795/9788447231003.013

Pp.: 274-291 


\section{Introducción: contexto docente}

La asignatura a la que se ha aplicado el ciclo de mejora es "Metodología y enseñanza de la lengua y literatura italiana" del cuarto año de la mención de Italiano que puede cursar el alumnado de diferentes grados de la Facultad de Filología. Se estructura en 6 créditos y en ella suelen matricularse un buen número de Erasmus italianos que provienen de Facultades de Letras y/o Lenguas. Este año, debido a la emergencia sanitaria, las personas provenientes de Italia han disminuido mucho y las personas que se han matriculado en el año académico 2020-2021 son solo 5 (2 alumnas de Filología hispánica, 1 alumno de Estudios Árabes e Islámicos y dos alumnas Erasmus italianas). La asignatura se imparte en 3 clases semanales de 80 minutos.

\section{Diseño previo del Ciclo de Mejora en el Aula (CIMA)}

El CIMA se ha desarrollado durante la 4áa-6a semana de clase (8 horas en total) y se ha organizado en 6 diferentes fases:

1. Plantear un problema general relacionado con la enseñanza de la lengua/literatura italiana.

2. Recoger las ideas previas del alumnado a través de un cuestionario

3. Crear subproblemas e intentar resolverlos con el apoyo de materiales y a través de una investigación personal y grupal

4. Diseñar una serie de actividades de contraste para resolver los subproblemas o contestar a las preguntas

5. Presentación de la propuesta didáctica de manera individual y actividad de feedback

6. Cuestionario final para poder evaluar el proceso de aprendizaje del alumnado intentando individuar las dificultades encontradas durante el ciclo de mejora y los recursos mas útiles para superarlas. 
Desde la primera clase se ha intentado plantear la asignatura de manera teórico-practica, debatiendo los diferentes contenidos y proponiendo actividades para explicitar los temas tratados. Este acercamiento ha permitido crear desde el principio un buen clima de trabajo en el que cada persona ha mostrado interés por la disciplina, aportando su punto de vista, compartiendo su experiencia previa como discente y, en algunos casos, como docente.

El problema/pregunta inicial que se ha propuesto ha sido: cómo crear una actividad didáctica de lengua o literatura para una clase de Italiano L2. Se ha imaginado un contexto concreto: que las/os participantes en la clase han sido designado para ocupar un puesto en una Escuela de Idioma o una Escuela Secundaria con la obligación de empezar en breve la actividad docente. Esta situación ha generado cuatro grandes subproblemas.

1) ¿Que me preocupa?

2) ¿Que quiero enseñar?

3) ¿Cómo quiero enseñar?

4) ¿Como evalúo?

\section{Mapa de contenidos}

La asignatura se ha organizado entorno a 4 preguntas fundamentales que están relacionadas entre ellas (Figura 1). Se trata de cuestiones que en mi opinión abarcan aspectos imprescindibles de la docencia. En la línea de lo que observa entre otros Balboni (2014) mi función ha intentado ser al mismo tiempo la de facilitador del aprendizaje, consejero/mayéutico/tutor y director que entre los bastidores intenta guiar el alumnado. Las diferentes cuestiones

Ciclos de Mejora en el Aula (2020). Experiencias de Innovación Docente de la US Esta obra se distribuye con la licencia Creative Commons 
(Figura 2-3-4-5) se han analizado y profundizado en las sesiones del ciclo de mejora.

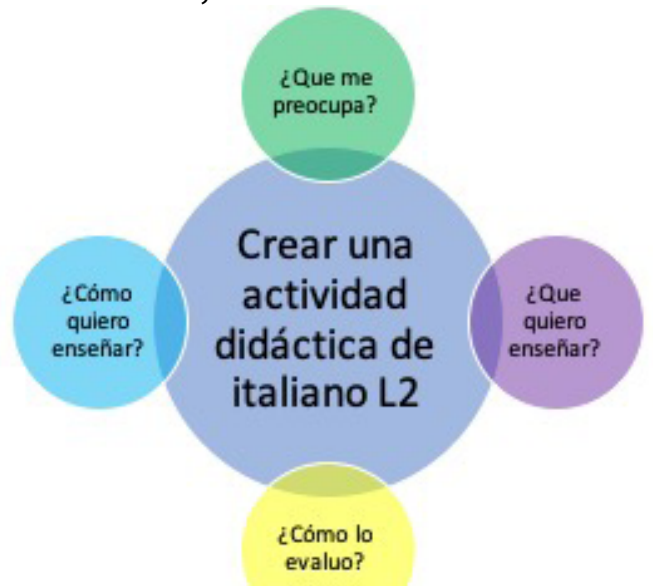

Figura 1. Mapa de contenido general de la asignatura

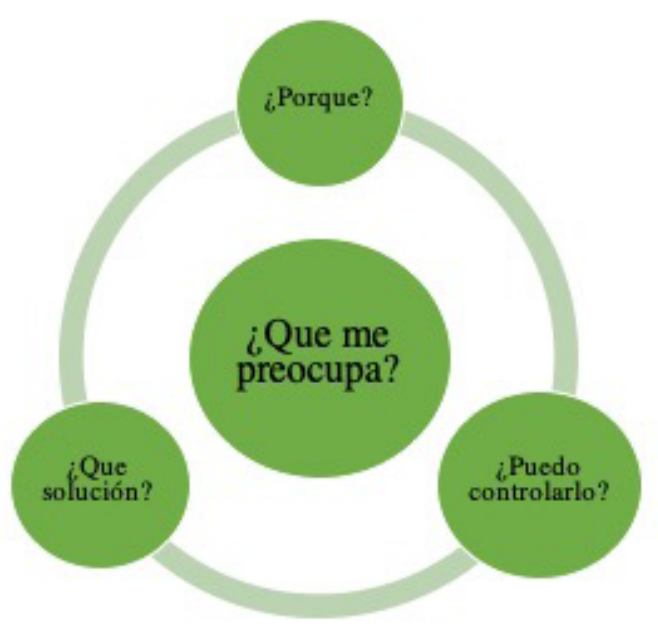

Figura 2. Mapa de contenido subproblema 1

Ciclos de Mejora en el Aula (2020). Experiencias de Innovación Docente de la US (c) (i) E Esta obra se distribuye con la licencia Creative Commons 


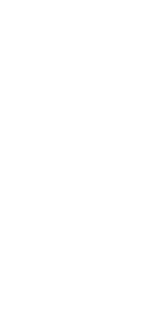

Figura 3. Mapa de contenido subproblema 2

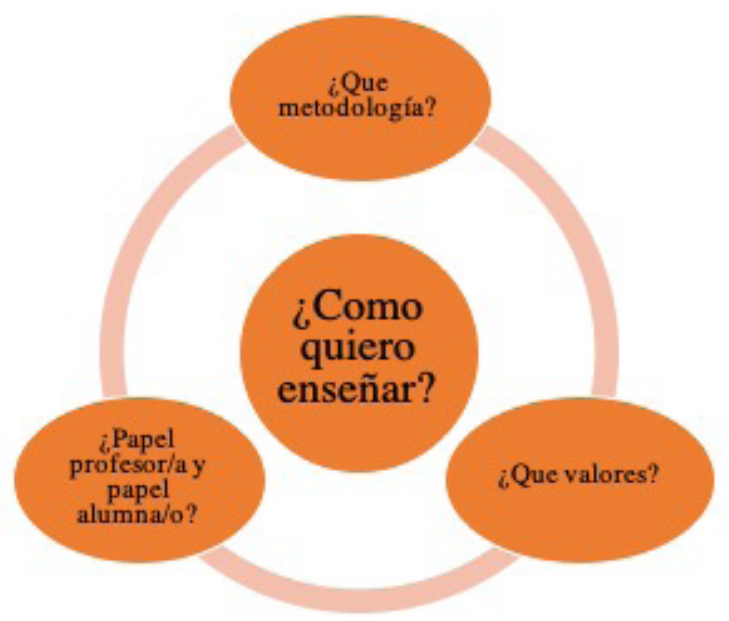

Figura 4. Mapa de contenido subproblema 3

Ciclos de Mejora en el Aula (2020). Experiencias de Innovación Docente de la US (c) (1) $\odot$ Esta obra se distribuye con la licencia Creative Commons 
¿Cuando

evaluo?

Figura 5. Mapa de contenido subproblema 4

Ciclos de Mejora en el Aula (2020). Experiencias de Innovación Docente de la US cc (i) $\odot$ Esta obra se distribuye con la licencia Creative Commons 
Modelo metodológico posible y secuencias de actividades programadas

Para el ciclo de mejora, entre las metodologías de enseñanza que señalan De Alba y Porlán (2017), se ha utilizado un modelo basado en el planteamiento de un problema inicial $(\mathrm{Pr})$ que ha permitido destacar las ideas previas del alumnado (la) que se han contrastado a través de diferentes actividades (Ac) y una práctica (P).

\section{PR}

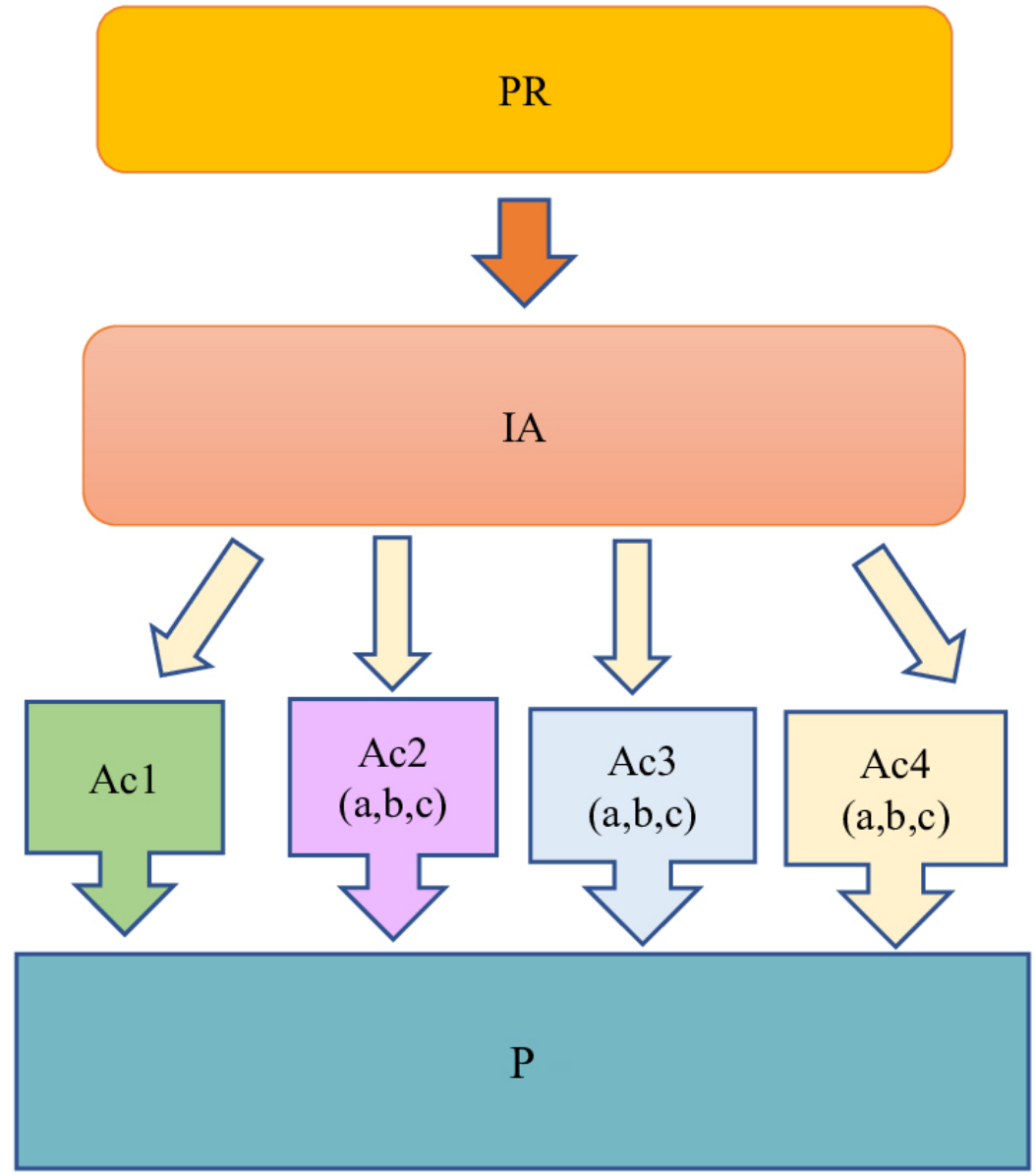

Figura 6. Modelo metodológico utilizado

Ciclos de Mejora en el Aula (2020). Experiencias de Innovación Docente de la US Esta obra se distribuye con la licencia Creative Commons 
En las siguientes tablas se recogen las diferentes actividades que se realizaron durante las sesiones del Ciclo de mejora.

\section{Tabla 1. Secuencia de actividades del ciclo de mejora}

\begin{tabular}{|l|l|l|}
\hline \multicolumn{3}{|l|}{ Sesión 1. Presentación del problema } \\
\hline Tipo de actividad & Descripción de la actividad & Tiempo \\
\hline $\begin{array}{l}\text { Planteamiento del } \\
\text { problema inicial }\end{array}$ & $\begin{array}{l}\text { Reflexión grupal sobre la cuestión } \\
\text { propuesta a la clase y elección } \\
\text { individual del tema a investigar } \\
\text { según los intereses personales }\end{array}$ & 10 minutos \\
\hline Cuestionario inicial & $\begin{array}{l}\text { Cuestionario de ideas previas } \\
\text { realizado a través de la Plataforma } \\
\text { Padlet }\end{array}$ & 20 minutos \\
\hline Actividad de debate & $\begin{array}{l}\text { Puesta en común y reflexión grupal } \\
\text { sobre las ideas previas }\end{array}$ & 20 minutos \\
\hline $\begin{array}{l}\text { Actividad de contraste } \\
\text { "Video Ken Robinson } \\
\text { "Bring on the learning } \\
\text { revolution" }\end{array}$ & $\begin{array}{l}\text { Video Ken Robinson (Ted, } \\
\text { Technology Entertainment Design) } \\
\text { subtitulado en italiano y debate } \\
\text { sobre conceptos e ideas principales } \\
\text { y más sugerentes del vídeo }\end{array}$ & 30 minutos \\
\hline
\end{tabular}

Sesión 2. Problema: Preocupaciones didácticas y primeros auxilios

\begin{tabular}{|l|l|l|}
\hline Tipo de actividad & Descripción de la actividad & Tiempo \\
\hline $\begin{array}{l}\text { Introducción sobre el } \\
\text { tema }\end{array}$ & $\begin{array}{l}\text { Presentación del tema con } \\
\text { referencias a la experiencia personal } \\
\text { del docente }\end{array}$ & 15 minutos \\
\hline $\begin{array}{l}\text { Actividad de contraste } \\
\text { 2a: }\end{array}$ & $\begin{array}{l}\text { Lectura artículo: “I ragazzi hanno } \\
\text { bisogno di insegnanti coraggiosi” y } \\
\text { reflexión sobre las ideas principales } \\
\text { del texto }\end{array}$ & 25 minutos \\
\hline $\begin{array}{l}\text { Actividad de contraste } \\
\text { 2b: }\end{array}$ & $\begin{array}{l}\text { Lectura artículo: “El primer día de } \\
\text { clases" y reflexión sobre las ideas } \\
\text { principales del texto }\end{array}$ & 25 minutos \\
\hline Reflexión final & $\begin{array}{l}\text { Debate final sobre preocupaciones } \\
\text { didácticas, auxilios y recursos }\end{array}$ & 15 minutos \\
\hline
\end{tabular}

Sesión 3. Problema: ¿Qué quiero enseñar y como lo quiero enseñar?

\begin{tabular}{l|l|l}
\hline Tipo de actividad & Descripción de la actividad & Tiempo
\end{tabular}

Ciclos de Mejora en el Aula (2020). Experiencias de Innovación Docente de la US Esta obra se distribuye con la licencia Creative Commons 


\begin{tabular}{|l|l|l|}
\hline $\begin{array}{l}\text { Modelos tradicionales } \\
\text { y modelos } \\
\text { innovadores }\end{array}$ & $\begin{array}{l}\text { Reflexión grupal sobre palabras claves } \\
\text { relacionadas con modelos didácticos } \\
\text { tradicionales e innovadores (Ver } \\
\text { figura 7 y figura 8) }\end{array}$ & \\
\hline $\begin{array}{l}\text { Actividad de contraste } \\
\text { 3a: }\end{array}$ & $\begin{array}{l}\text { Lectura y análisis grupal de la tabla } \\
\text { "Approcci e metodi" (desde "Le sfide } \\
\text { di Babele" di P. Balboni Ed. Utet } \\
\text { Università) ¿En qué modelos me } \\
\text { reconozco? ¿Cómo quiero trabajar los } \\
\text { contenidos que quiero proponer? }\end{array}$ & 20 minutos \\
\hline $\begin{array}{l}\text { Actividad de contraste } \\
\text { 3b: }\end{array}$ & $\begin{array}{l}\text { Presentación, análisis de algunas } \\
\text { propuestas didácticas con referencias } \\
\text { a las propuestas iniciales del } \\
\text { alumnado }\end{array}$ & 20 minutos \\
\hline $\begin{array}{l}\text { Actividad de contraste } \\
\text { 3c: }\end{array}$ & $\begin{array}{l}\text { Taller con actividades y práctica de } \\
\text { herramientas útiles para desarrollar } \\
\text { las diferentes propuestas didácticas }\end{array}$ & 25 minutos \\
\hline
\end{tabular}

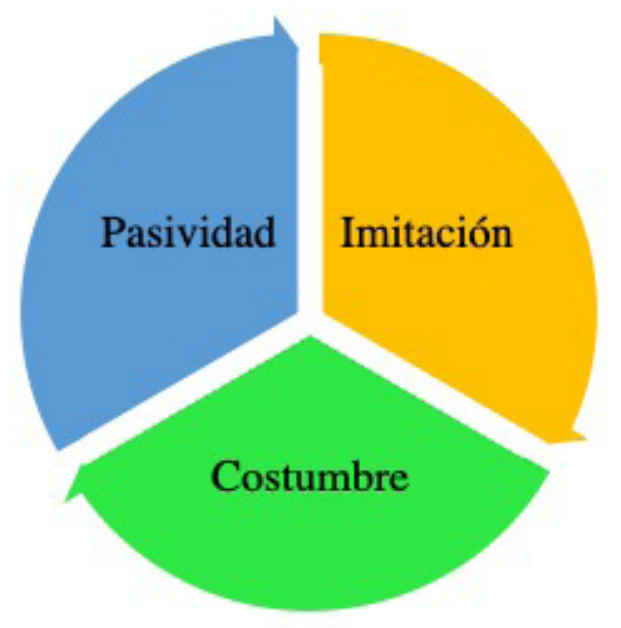

Figura 7. Características modelos didácticos tradicionales

Ciclos de Mejora en el Aula (2020). Experiencias de Innovación Docente de la US Esta obra se distribuye con la licencia Creative Commons 


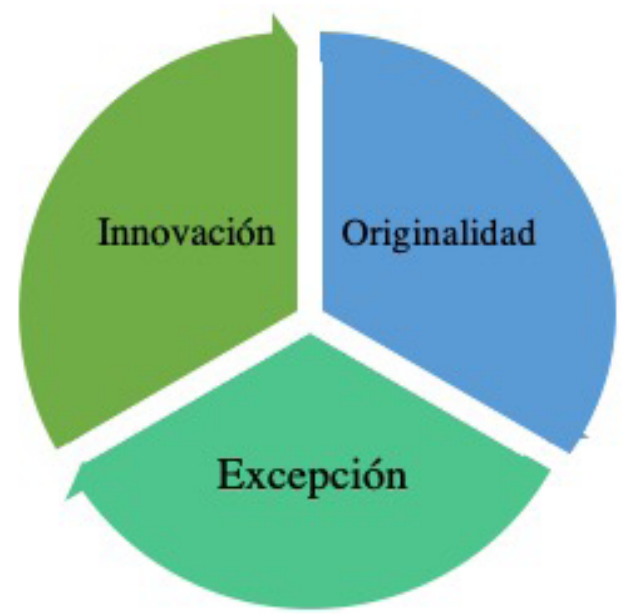

Figura 8. Características modelos didácticos innovadores

\begin{tabular}{|l|l|l|}
\hline \multicolumn{2}{|l|}{ Sesión 4. Problema: ¿Cómo evalúo? } \\
\hline Tipo de actividad & Descripción de la actividad & Tiempo \\
\hline $\begin{array}{l}\text { Actividad de } \\
\text { contraste 4a }\end{array}$ & $\begin{array}{l}\text { Exámenes de película: visión de } \\
\text { breves fragmentos de películas } \\
\text { italianas relacionadas con la } \\
\text { evaluación y los exámenes y } \\
\text { reflexión grupal }\end{array}$ & 20 minutos \\
\hline $\begin{array}{l}\text { Actividad de } \\
\text { contraste 4b: }\end{array}$ & $\begin{array}{l}\text { Lectura del texto “La valutazione” } \\
\text { desde Alma Edizioni y reflexión } \\
\text { sobre las diferentes pruebas de } \\
\text { evaluación }\end{array}$ & 30 minutos \\
\hline $\begin{array}{l}\text { Actividad de } \\
\text { contraste 4c: }\end{array}$ & $\begin{array}{l}\text { La evaluación, cómo, cuándo y } \\
\text { porqué. Taller práctico }\end{array}$ & 30 minutos \\
\hline
\end{tabular}

\begin{tabular}{|l|l|l|}
\hline \multicolumn{2}{|l|}{ Sesión 5. Practica y cuestionario final } \\
\hline Tipo de actividad & Descripción de la actividad & Tiempo \\
\hline Práctica & $\begin{array}{l}\text { Presentación individual de una } \\
\text { actividad sobre el tema elegido }\end{array}$ & 60 minutos \\
\hline $\begin{array}{l}\text { Feedback y } \\
\text { evaluación: }\end{array}$ & $\begin{array}{l}\text { Comentarios y debate sobre las } \\
\text { actividades propuestas }\end{array}$ & 30 minutos \\
\hline Cuestionario final & $\begin{array}{l}\text { Cuestionario final realizado a través } \\
\text { de la Plataforma Padlet }\end{array}$ & 20 minutos \\
\hline
\end{tabular}

Ciclos de Mejora en el Aula (2020). Experiencias de Innovación Docente de la US Esta obra se distribuye con la licencia Creative Commons 
Cuestionario previo y final

A continuación, se indican las preguntas que se presentaron a través de Padlet al alumnado al principio y al final del Ciclo de Mejora

1) ¿Cuáles son tus principales preocupaciones al momento de realizar una propuesta didáctica?

2) ¿Cuáles son los principios didácticos mas importantes que quieres trasmitir?

3) ¿Qué metodología piensas que sea la mas adecuada para desarrollar los contenidos de tu propuesta?

4) ¿Cuál metodología piensas utilizar para evaluar el proceso de aprendizaje de tu clase?

\section{Aplicación del Ciclo de mejora}

\section{Resumen de las sesiones}

\section{Sesión1}

La primera sesión del Cima ha sido importante para crear un clima de trabajo ideal y permitir que el alumnado se sintiera cómodo y con la posibilidad de expresar líberamente las propias opiniones, creencias y preocupaciones. La elección de un problema práctico (el nombramiento por parte de una escuela de idioma) ha permitido situar la clase en un contexto real e identificarse en el papel docente. Las preguntas presentadas giraban entorno a cuatro cuestiones generales, que han caracterizado las siguientes sesiones. La actividad de contraste propuesta (el vídeo de Ken Robinson) ha ofrecido muchos elementos inspiradores para poder seguir con el debate y la reflexión. 
Sesión 2

En todas las fases del Cima, pero especialmente en esta sesión, considero que ha sido fundamental compartir mi experiencia personal como docente e intentar trasmitir las sensaciones vividas en diferentes situaciones. Los dos artículos propuestos como actividades de contraste han ofrecido la posibilidad de analizar una cuestión fundamental como el papel del docente y una situación concreta como el primer día de clases. Se ha generado un proficuo debate y un recíproco intercambio de ideas durante la reflexión final.

\section{Sesión 3}

Empezar la sesión trabajando sobre palabras claves ha dado la posibilidad de visualizar algunos de los conceptos que se querían abarcar a lo largo del Cima. El análisis de la tabla "Approcci e metodi" ha permitido al alumnado sintetizar de manera eficaz diferentes metodologías didácticas y centrarse en los aspectos que querían utilizar por la propia actividad didáctica. El análisis critica de otras propuestas didácticas ha permitido observar y evaluar estrategias y soluciones utilizadas por otros docentes y personal investigador. El taller final dedicado a algunas herramientas didácticas online (plataformas, gamificación etc..) ha ofrecido una panorámica sobre las posibilidades que ofrece la web para completar e implementar la docencia.

\section{Sesión 4}

La visión de algunos breves fragmentos de películas que trataban de la evaluación y de los exámenes ha permitido introducir el tema objeto de la sesión de manera amena. El análisis del artículo sobre la evaluación ha hecho hincapié en un ejemplo utilizado para la enseñanza del Italiano L2 y permitido considerar los aspectos de este tipo de evaluación que se podrían adoptar y cuáles sería necesario adaptar. La última parte de la sesión, dedicada a las varias fases y momentos de la evaluación, ha sido útil 
para pasar en reseña diferentes aspectos y metodologias. La posibilidad de observar diferentes herramientas desde las perspectivas del alumnado y como futuras/os docentes creo que ha sido muy enriquecedora.

\section{Sesión 5}

La exposición por parte del alumnado de la propuesta didáctica que habían trabajado a lo largo del Cima ha sido un momento muy satisfactorio. He sido un alumno más del grupo y he participado en las varias actividades realizadas, "dando clase con boca cerrada" (Finkel, 2008). A través de su presentación cada persona ha podido experimentar cuestiones y problemáticas que se habían analizado y tratado a través de las diferentes actividades de contraste. La modalidad online ha representado un valor añadido a la experiencia didáctica del alumnado, porque se han podido introducir nuevas dinámicas y metodologías como se ha destacado en los comentarios y en el debate final.

\section{Evaluación del aprendizaje del alumnado}

Para evaluar el aprendizaje del alumnado se han tenido en cuenta el cuestionario inicial y final, los varios comentarios producido durante las diferentes actividades, la asistencia y también las aportaciones en el Padlet de la asignatura fuera del horario de clase.

Cuestión 1: ¿Cuáles son tus principales preocupaciones en el momento de realizar una propuesta didáctica?

Las principales preocupaciones que se ha señalado antes de la aplicación del Cima han sido bastante generales:

a) que el tema no sea interesante y motivador

b) no poder controlar diferentes aspectos de la docencia (organización actividades, diferentes niveles del alumnado, tener un plan alternativo).

Ciclos de Mejora en el Aula (2020). Experiencias de Innovación Docente de la US Esta obra se distribuye con la licencia Creative Commons 
Analizando las respuestas, después de la aplicación del Cima, se puede destacar que las preocupaciones se centran en aspectos mas concretos y se han detallado, demostrando una mayor conciencia y conocimiento de la disciplina. Se señalaban, por ejemplo:

a) la importancia de encontrar un nivel de dificultad que se adapte a todo el alumnado

b) individualizar la docencia, infravalorar o subvalorar los conocimientos previos de la clase

c) no recibir feedback,

d) no aprovechar al máximo las herramientas de interacción que el aula (virtual) proporciona.

Cuestión 2: ¿Cuáles son los principios didácticos mas importantes que quieres trasmitir?

En este caso los principios didácticos aparecían ya en parte definidos, porque en las clases anteriores a la aplicación del Cima ya se había reflexionado sobre diferentes sistemas educativos. Se señalaban, por ejemplo:

a) la importancia de superar una enseñanza basada en teorías y nociones a favor de un aprendizaje mas creativo,

b) la necesidad de incentivar la motivación y la autonomía del discente.

Después de la aplicación del Cima la atención se ha centrado también en otros aspectos que han hecho hincapié en las diferentes propuestas didácticas, como:

a) a importancia de trabajar en equipo y de impulsar la colaboración

b) la ayuda entre compañeras/os,

c) el aprendizaje lúdico,

d) la conexión entre teoría y practica,

e) la eficacia de una metodología como las flipped classroom,

f) enseñar a superar el miedo al error y verlo como una oportunidad. 
Cuestión 3: ¿Qué metodología piensas que son las mas adecuadas para desarrollar los contenidos de tu propuesta?

Entre las metodologías indicada al principio del Cima, las que parecían la más adecuadas resultaban ser la metodología participativa y cooperativa. Después de la aplicación del Cima y de la práctica, los mismos métodos seguían siendo importantes, pero se destacaba la necesidad de variar entre actividad individual y colectiva, para que cada persona pudiera construir su propio aprendizaje y plantearse retos. Contemporáneamente se subrayaba la importancia de los recursos digitales, que si bien aplicados, pueden facilitar y mejorar la calidad de la docencia.

Cuestión 4: ¿Cuál metodología piensas utilizar para evaluar el proceso de aprendizaje de tu clase?

Si en el cuestionario inicial, en parte, ya se hacía referencia a la necesitad de métodos que no intimidaran al alumnado y a una evaluación continua, después del Cima, se ha manifestado la posibilidad de evaluar también basándose en una investigación realizada por el alumnado, siguiendo los intereses personales o profundizando un tema especifico de la asignatura. La evaluación podría consistir en un portafolio con varias tareas puntuales que se entregan durante el desarrollo de las clases.

\section{Evaluación del CIMA puesto en practica}

La aplicación del Cima me ha permitido analizar más en profundidad la enseñanza de la lengua y literatura italiana. Se ha tratado de un proceso que he llevado a cabo junto al alumnado y sus consideraciones y comentarios han enriquecido notablemente mi reflexión y mi proceso de aprendizaje. La participación y la implicación de la clase ha sido muy satisfactoria y la utilización de una aplicación como el Padlet ha sido acertada, porque ha permitido un seguimiento y monitoraje del Cima fuera de las horas de clase. Se han subido a la plataforma virtual los materiales

Ciclos de Mejora en el Aula (2020). Experiencias de Innovación Docente de la US Esta obra se distribuye con la licencia Creative Commons 
utilizados para que las personas pudieran volver a analizarlos y añadieran comentarios y nuevos contenidos. Considero que la metodología aplicada ha funcionado bien y se podría ampliar a buena parte de la asignatura, diseñando un futuro Ciclo de Mejora más amplio. El objetivo principal del Cima que consistía en desarrollar un pensamiento critico del alumnado entorno a la disciplina se ha realizado Con respeto al cuestionario proporcionado, quizás en vista de futuras aplicaciones, una alternativa podría consistir en formular las preguntas de manera diferente, proponiendo casos prácticos para poder realizar un análisis más cuantitativo y no solo cualitativo. Finalmente describir y valorar las diferentes fases de mi ciclo de mejora a utilizando un diario de clase (Porlan, 2008) me ha ayudado a ser mas consciente de mi papel como docente, a concretar mejor mis ideas y programar con mayor precisión mi intervención.

\section{Principios didácticos fundamentales del CIMA}

Entre los principios didácticos que han guiado la elaboración de mi ciclo de mejora que considero fundamentales para mi modelo didáctico personal (García Pérez y Porlán, 2017), quiero señalar los siguientes:

- Para conseguir una mayor implicación del alumnado es importante tomar como punto de partida un problema y definirlo como una situación real. Si los contenidos están relacionados con cuestiones relevantes de la realidad será mas fácil destacar su utilidad y despertar el interés.

- Analizar y reflexionar sobre los modelos mentales del alumnado es imprescindible para poder planificar nuestra docencia, realizar eventuales cambios en los contenidos y adaptar la secuencia de actividades que queremos proponer.

- El alumnado tienes que figurar constantemente en el centro del proceso de aprendizaje. Así podrá construir

Ciclos de Mejora en el Aula (2020). Experiencias de Innovación Docente de la US Esta obra se distribuye con la licencia Creative Commons 
su conocimiento con la orientación del docente. Es necesario trabajar no solo los contenidos conceptuales sino también aspectos como la participación crítica, contrastando diferentes puntos de vista.

- Es esencial reflexionar constantemente sobre nuestra docencia. Autoevaluarse y ser evaluado nos puede ayudar a una mayor consciencia de nuestra labor y a entender mejor las necesidades del alumnado.

Palabras clave: Metodología de la enseñanza de la lengua y literatura italiana, filología, docencia universitaria, experimentación docente universitaria.

Key words: Methodology and teaching of Italian language and literature, philology, university teaching, university teaching experimentation.

Ciclos de Mejora en el Aula (2020). Experiencias de Innovación Docente de la US Esta obra se distribuye con la licencia Creative Commons 


\section{Referencias bibliográficas}

Alma Edizioni. (2015). La valutazione.Officina.it. Recuperado de https://www.almaedizioni.it/it/officina/2015/ officina-25/

Balboni, P. E. (2014). Didattica dell'italiano come lingua straniera, Torino: Bonacci Editore.

Balboni, P. E. (2002). Approcci e metodi. En P. E. Balboni, Le sfide di Babele. Insegnare le lingue nelle società complesse, Torino: Utet Libreria. Recuperado de http:// www00.unibg.it/dati/bacheca/497/23782.pdf

Cicero, N. K. (2018). El primer día de clases, Revista de educación y derecho, 18. Recuperado de https:// revistes. ub.edu/index.php/RED/article/view/22927

De Alba, N. y Porlán, R. (2017). La metodología de enseñanza. En R. Porlán (Ed.), Enseñanza Universitaria. Cómo mejorarla (pp. 37-53). Madrid: Editorial Morata.

Finkel, D. (2008). Dar clase con la boca cerrada. Valencia: Universidad de Valencia.

Forster, L. (2016). I ragazzi hanno bisogno di insegnanti coraggiosi, Internazionale. Recuperado de https://www.internazionale.it/opinione/lizanne-foster/2016/01/03/ scuola-insegnanti

García Pérez, F. F. y Porlán, R. (2017) Los Principios Didácticos y el Modelo Didáctico Personal. En R. Porlán (Ed.), Enseñanza Universitaria. Cómo mejorarla (pp. 93-104). Madrid: Editorial Morata.

Porlán, R. (2008). El diario de clase y el análisis de la práctica, Averroes. Red Telemática Educativa de Andalucía. Recuperado de https://idus.us.es/handle/11441/25448

Ciclos de Mejora en el Aula (2020). Experiencias de Innovación Docente de la US Esta obra se distribuye con la licencia Creative Commons 